AIP $\mid \begin{aligned} & \text { Joumalof } \\ & \text { Applied Physics }\end{aligned}$

\title{
Surface aided polarization reversal in small ferroelectric particles
}

K.-H Chew, J. Osman, R. L. Stamps, D. R. Tilley, F. G. Shin et al.

Citation: J. Appl. Phys. 93, 4215 (2003); doi: 10.1063/1.1558203

View online: http://dx.doi.org/10.1063/1.1558203

View Table of Contents: http://jap.aip.org/resource/1/JAPIAU/v93/i7

Published by the AIP Publishing LLC.

\section{Additional information on J. Appl. Phys.}

Journal Homepage: http://jap.aip.org/

Journal Information: http://jap.aip.org/about/about_the_journal

Top downloads: http://jap.aip.org/features/most_downloaded

Information for Authors: http://jap.aip.org/authors

\section{ADVERTISEMENT}

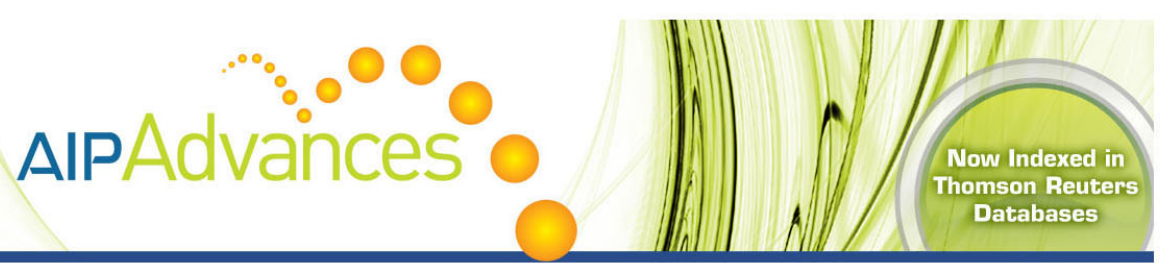

\section{Explore AIP's open access journal: Rapid publication \\ - Article-level metrics \\ - Post-publication rating and commenting}




\title{
Surface aided polarization reversal in small ferroelectric particles
}

\author{
K.-H Chew ${ }^{\mathrm{a})}$ and J. Osman ${ }^{\mathrm{b}}$ ) \\ School of Physics, Universiti Sains Malaysia, 11800 Penang, Malaysia \\ R. L. Stamps \\ School of Physics, University of Western Australia, 35 Stirling Highway, Crawley, WA 6009, Australia \\ D. R. Tilley \\ School of Physics, Universiti Sains Malaysia, 11800 Penang, Malaysia \\ F. G. Shin and H. L. W. Chan \\ Department of Applied Physics, The Hong Kong Polytechnic University, Hung Hom, Kowloon, Hong Kong
}

(Received 9 October 2002; accepted 13 January 2003)

\begin{abstract}
Polarization reversal in ferroelectric particles driven by a pulsed electric field is examined theoretically using Landau-Devonshire-Khalatnikov theory. A significant reduction in reversal times is shown to be possible if certain surface properties and size criteria are met. The surface properties are also shown to control the magnitude of the applied field needed for irreversible switching. An interesting signature of surface effects is found in the switching current. The theory predicts that the switching current for small ferroelectric particles can exhibit double peaks as a function of time. The size and relative times of the peaks provide specific information on the magnitude and rate of surface reversal dynamics. (c) 2003 American Institute of Physics.
\end{abstract}

[DOI: $10.1063 / 1.1558203$ ]

Polarization reversal in mesoscopic ferroelectric structures is a topic of increasing interest, particularly in view of the rapid developments in fabrication technologies. Nanosized ferroelectric elements and patterned media are challenging systems for the study of fundamental problems in ferroelectric to provide key technological solutions for highdensity ferroelectric memory devices. ${ }^{1}$ Methods such as electron-beam lithography, ${ }^{2}$ focused ion-beam milling, ${ }^{3}$ and self assembly ${ }^{4}$ are now standard techniques for producing high-quality arrays of nanometer scale ferroelectric particles. ${ }^{5-8}$

Mesoscopic structures of small ferroelectric particles are very interesting from a fundamental research point of view. Their typical dimensions are comparable with the characteristic length $\xi_{0}$ of the materials, so they can show new phenomena. ${ }^{5-7}$ An example of the importance of surface conditions on polarization properties can be seen from two recent experiments. In one case, an asymmetrical piezoelectric hysteresis loop was observed for polycrystalline lead zirconate titanate (PZT) mesoscopic structures. ${ }^{5}$ The asymmetrical property of the loop was found to be dependent on size and due to the pinning of nonswitching domains. A separate study on single-crystal $\mathrm{PZT}^{6}$ showed that the hysteresis was dominated by the depinning of domains caused by the crystalline structure of the surfaces. It is clear from these two examples that the microscopic structure of the surfaces can dominate ferroelectric ordering in small particles.

While extensive theoretical studies of polarization reversal ${ }^{7,9-10}$ have been made for bulk and thick film geometries, very little work has been performed for reversal in nanoscale geometries in which the surface conditions can

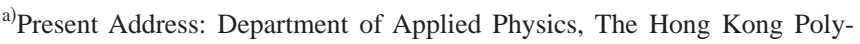
technic University, Hung Hom, Kowloon, Hong Kong.

${ }^{b}$ Electronic mail: junaidah@usm.my
}

dominate over the bulk. Ghosez and Rabe ${ }^{11}$ in their microscopic study pointed out that ferroelectric film of thickness in the nanometer range shows a significant enhancement of polarization at the surface. The purpose of the present article is to show how such enhancements can dramatically influence the dynamic switching behavior.

We consider a one-dimensional model with polarization and related physical quantities varying as a function of $x$. The discussion is restricted to in-plane geometries where the thickness is much less than the lateral dimensions so that depolarization effects can be neglected. A small ferroelectric particle of a cross-sectional area $S$ in the $y-z$ plane and length $L$ in the $x$ direction is assumed. The LandauDevonshire free energy per unit area for a second-order ferroelectric under an applied electric-field $E$ is

$F=\frac{1}{2} \frac{A\left(T-T_{C}\right)}{\varepsilon_{0} S^{2}} P^{2}+\frac{1}{4} \frac{B}{\varepsilon_{0}^{2} S^{4}} P^{4}+\frac{1}{2} \frac{D}{\varepsilon_{0} S^{2}}\left(\frac{d P}{d x}\right)^{2}-E \frac{1}{S} P$,

where $A$ and $B$ are temperature-independent parameters. For a second-order transition, $B$ is a positive value and $T_{C}$ is the Curie temperature of the material. The polarization $P$ is defined as the dipole moment per unit length $P=S Q, Q$ being the dipole moment per unit volume. The coefficient $D$ is associated with the spatial variation of $P$ along the $x$ direction. The surface and size effects on the reversal properties are studied by introducing the so-called extrapolation length $\delta$ in the boundary conditions ${ }^{12}$

$$
\frac{d P}{d x}= \pm \frac{P}{\delta} \quad \text { at } x=0 \quad \text { and } L .
$$

For a positive extrapolation length, the polarization is reduced at the surface. If the extrapolation length is negative, polarization is enhanced at the film surface. 
An understanding of how reversal occurs can be achieved by examining the phenomenological equation of motion used in the Landau-Devonshire-Khalatnikov theory as

$\gamma \frac{d P}{d \tau}=-\frac{d F}{d P}=-\frac{A\left(T-T_{C}\right)}{\epsilon_{0} S} P-\frac{B}{\varepsilon_{0}^{2} S^{3}} P^{3}+\frac{D}{\varepsilon_{0} S} \frac{d^{2} P}{d x^{2}}+E$,

where $\gamma$ is the viscosity coefficient which causes delay in domain wall movement.

In the calculations, we rescale the variables as follows: $t=T / T_{C} ; p=P / P_{0} ; \zeta=x / \xi_{0}$; and $s=S / \xi_{0}^{2} . P_{0}$ is the bulk polarization which we define as $\xi_{0}^{2}\left(-A T_{C} \varepsilon_{0} / 3 B\right)^{1 / 2}$, where $\xi_{0}=\left(D / A T_{C}\right)^{1 / 2}$ corresponds to the characteristic length of the material.

The normalized Landau-Devonshire-Khalatnikov equation of motion becomes

$$
\frac{d p}{d \tau^{\prime}}=(1-t) \frac{1}{s} p-\frac{1}{s^{3}} p^{3}+\frac{1}{s} \frac{d^{2} p}{d \zeta^{2}}+e,
$$

where the time variable is $\tau^{\prime}=\tau\left(a T_{C} / \gamma \varepsilon_{0} \xi_{0}^{2}\right)$ and the applied field variable $e=E\left(B \varepsilon_{0} / a^{3} T_{C}^{3}\right)^{1 / 2}$.

The boundary conditions are

$$
\frac{d p}{d \zeta}= \pm \frac{p}{\delta^{\prime}} \quad \text { at } \zeta=0 \quad \text { and } L / \xi_{0},
$$

with the rescaled form of extrapolation length as $\delta^{\prime}=\delta / \xi_{0}$. The minimum applied field required for polarization reversal for free boundary condition $(d p / d \zeta=0)$ in rescaled form becomes

$$
e_{c}= \pm \frac{2}{3}(1-t)\left(\frac{1-t}{3}\right)^{1 / 2}
$$

The polarization current resulting from the applied field $e$ is defined as

$$
i=\frac{d p}{d \tau^{\prime}} .
$$

The differential Eq. (4) was solved numerically using a finite-difference scheme subject to the boundary conditions of Eq. (5). In the example, results that follow, values appropriate for $\mathrm{BaTiO}_{3}$ were taken: ${ }^{13} A=5.9 \times 10^{-6} \mathrm{~K}^{-1}, B=1.9$ $\times 10^{-13} \mathrm{~m}^{3} \mathrm{~J}^{-1}$, and $D=1.0 \times 10^{-18} \mathrm{~m}^{2}$. The estimated value of domain wall width $\xi_{0}$ is $20.8 \mathrm{~nm}$ for $\mathrm{BaTiO}_{3}$. Unless otherwise specified, the values $S=4.0 \xi_{0}^{2}$ and $T=0.5 T_{C}$ are assumed.

We first examine the dynamics of reversal under a simple step function driving field $\phi$

$$
e\left(\tau^{\prime}\right)=\phi\left(\tau^{\prime}\right) e_{0} .
$$

The effect on switching is shown in Fig. 1 where the polarization across the film thickness plotted at different times for two types of surface conditions (a) $\delta=-2.0 \xi_{0}$ and (b) $\delta=$ $+2.0 \xi_{0}$. The initial polarization is set at the negative state. The result shows that in each case, (a) or (b), the switching of polarization near the surfaces and in the interior is initiated and completed at different times. A comparison with the
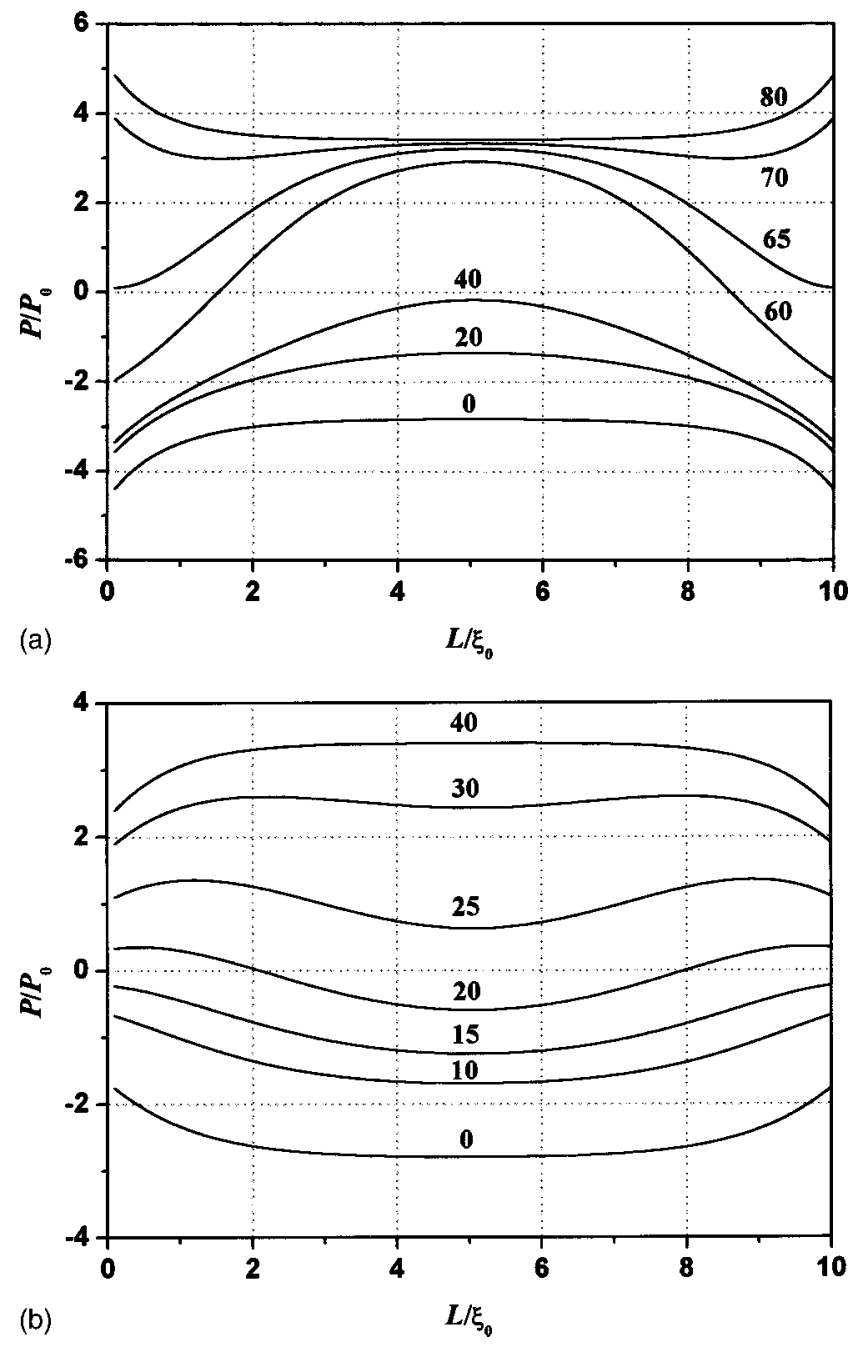

FIG. 1. Polarization across the thickness for different times during reversal under a step field. The field is applied at $\tau^{\prime}=0.0$ with magnitude $1.5 e_{c}$. The surface parameter $\delta$ is $-2.0 \xi_{0}$ for (a) and $+2.0 \xi_{0}$ for (b).

two surface conditions shows an overall longer time is required for a complete reversal for the surface with polarization enhanced near the surface.

We now consider the reversal dynamics under a pulsed field. The form of the pulse is assumed to be Gaussian with peak magnitude $e_{0}$ and a width specified by $\sigma$

$$
e\left(\tau^{\prime}\right)=e_{0} \exp \left[-\sigma \tau^{\prime 2}\right]
$$

The main difference between driving with a pulsed field as opposed to the step field is that the reversal now depends upon both the field strength and duration, or width, of the pulse. If the value of $e_{0}$ and $\sigma$ are too small, the reversal will not occur. In the present study, the pulse field is set so that the field reaches its maximum at $\tau^{\prime}=100.0$. The reversal process for the surface with enhanced polarization is shown in Fig. 2 for the surface and interior polarization as a function of time with $\delta=-2.0 \xi_{0}$. In Fig. 2(a), when the driving field is too small, the polarization will decrease toward zero rapidly at first, and then relax back to the initial state. Thus, the driving field should exceed a minimum field for reversal. The 


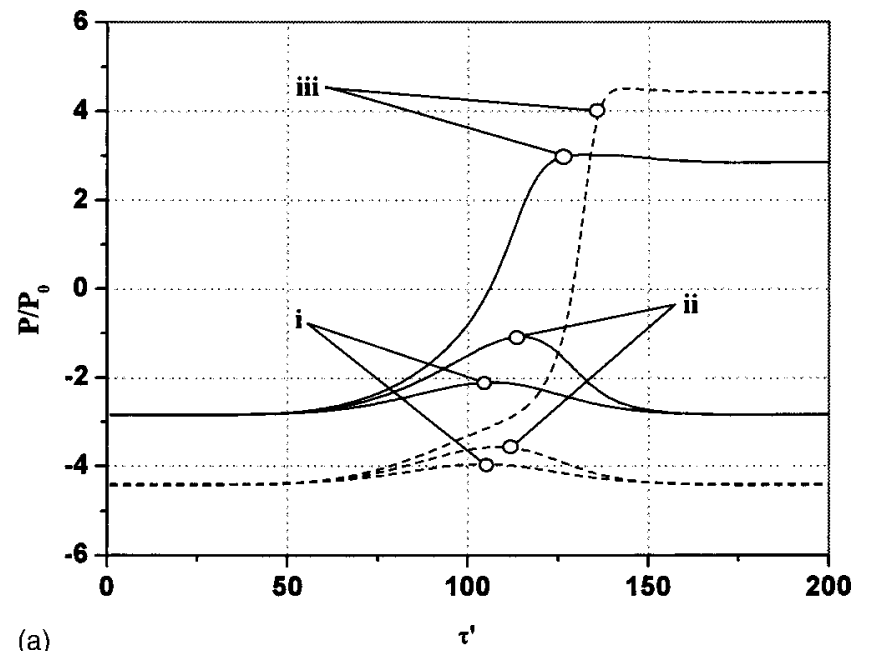

(a)

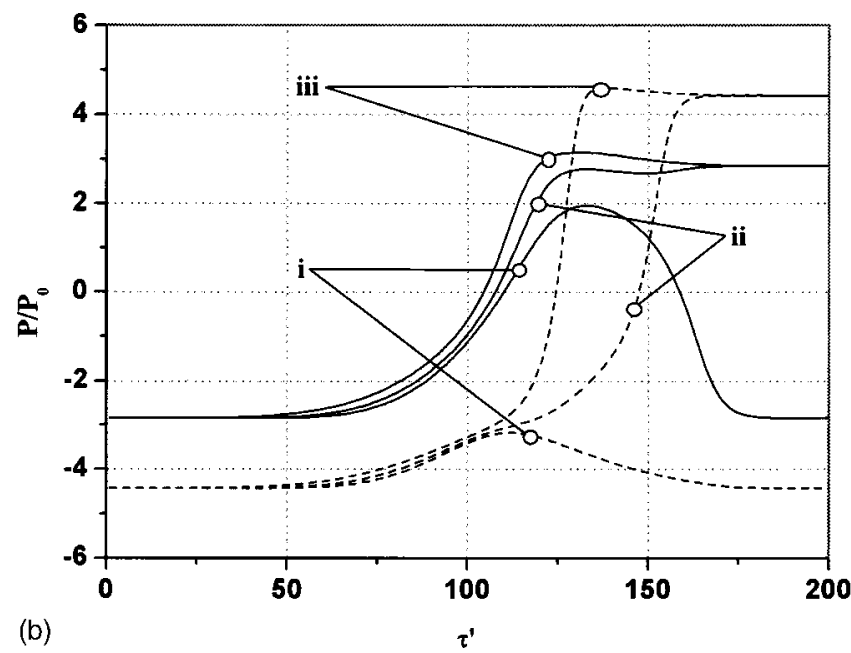

FIG. 2. Polarization as a function of time for different switching field strengths with $\delta=-2.0 \xi_{0}$ for thickness $L=10.0 \xi_{0}$. The switching field is a pulsed Gaussian field with pulse width (a) $\sigma=1000$ and field strength (i) $e_{0}=1.0 e_{C}$, (ii) $e_{0}=1.5 e_{C}$, and (iii) $e_{0}=1.8 e_{C}$. In (b), the field strength is $e_{0}=1.8 e_{C}$ and pulse width (i) $\sigma=600$, (ii) $\sigma=800$, and (iii) $\sigma=1200$. The solid lines show the polarization in the interior of the film, and the dotted lines show the polarization at the surfaces.

reversal mechanism is also strongly determined by the pulse width $\sigma$, and there is a minimum width for each field, as shown in Fig. 2(b).

Polarization enhancement near the surface results in different reversal times for the interior and surface. The reversal time is sensitive to the sign and magnitude of the surface parameter, and strongly affects the minimum reversal field $e_{\min }$. Figure 3 shows the minimum reversal field as a function of the surface parameter. The minimum switching field $e_{\min }$ is defined as the smallest driving field needed to bring both the polarization of the interior and surface from its initial negative state to positive. A large surface parameter value corresponds to a weak surface pinning effect and results in a smaller critical field. Similarly, the contribution of the surface conditions goes as inversely proportional to thickness, leading to a decrease in the minimum switching field with increasing thickness. As thickness increases, there is less surface effect and the minimum field needed for reversal approaches that of a bulk material.

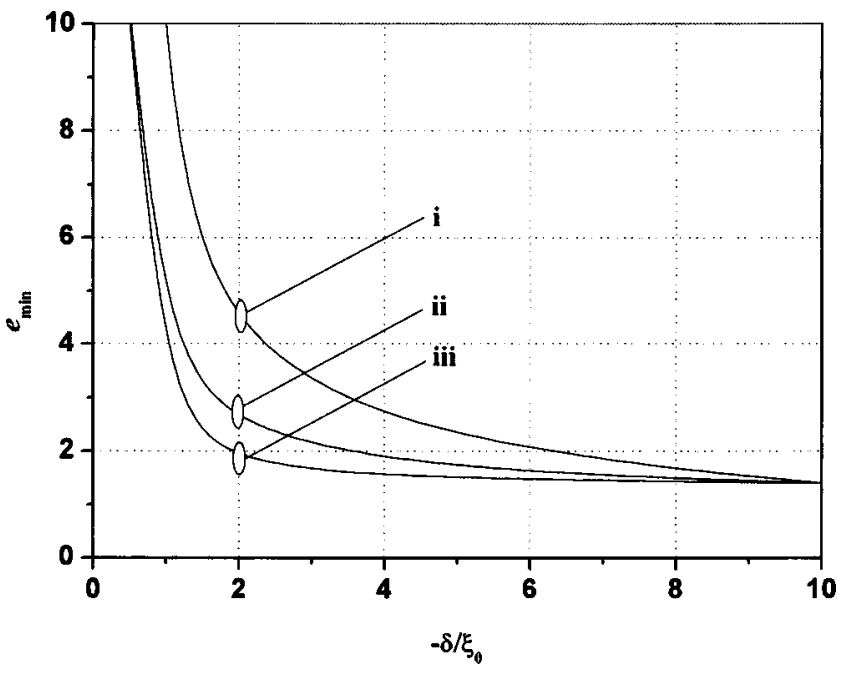

FIG. 3. Minimum switching field $e_{\min }$ as a function of surface parameter for different thicknesses. The switching field is a pulsed Gaussian field with pulse width $\sigma=1000$. In the curves, the thicknesses are (i) $L=2.0 \xi_{0}$, (ii) $L=5.0 \xi_{0}$, and (iii) $L=10.0 \xi_{0}$.

Extremely interesting features can be observed by examining the polarization current as a function of time for reversal which is driven by the Gaussian pulsed field of Eq. (9). Examples are shown in Fig. 4 where the total polarization current averaged over the entire thickness is shown as (i). The interior and surface polarization currents are shown separately as (ii) and (iii). The curves show how the reversal is signaled by a maximum in the polarization current, and that separate maxima occur for surface and interior contributions. The important feature is that these two maxima can be clearly distinguished in a measurement of the total polarization current, for what one might expect from an experimental measurement of switching.

In summary, we have shown how the surface ferroelectric polarization can strongly influence driven polarization

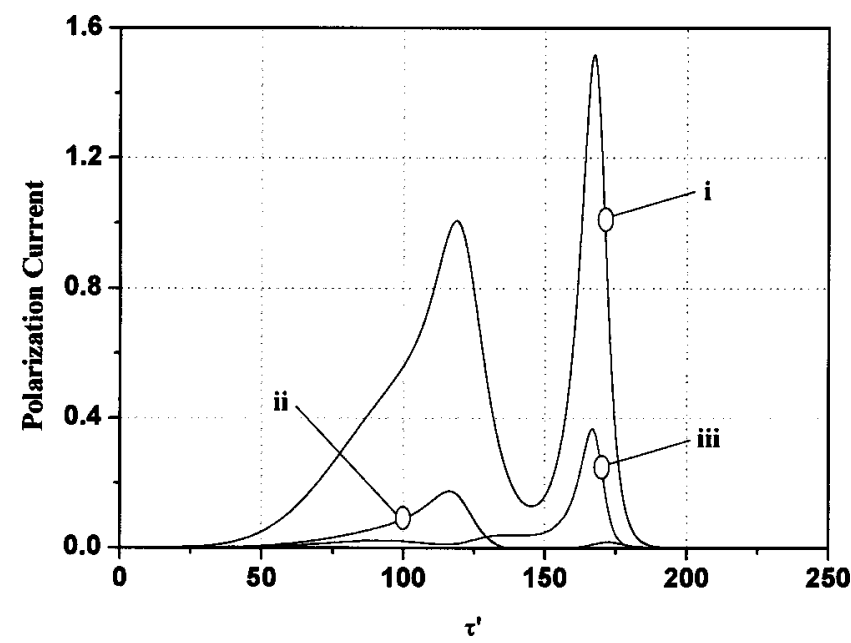

FIG. 4. Polarization current as a function of time during reversal under a Gaussian pulsed field for thickness $L=10.0 \xi_{0}$. The field strength and pulse width are $e_{0}=1.73 e_{c}$ and $\sigma=1000$. Curve (i) is the total reversal current averaged over the thickness $L$. The early peak is due to the interior polarization reversal which denotes as "interior-peak," shown by (ii), and the later corresponds to surface reversal or "surface-peak," indicated by (iii). 
reversal in small ferroelectric particles. If the surface polarization is enhanced relative to the interior, the reversal process is slowed. Two distinct reversal peaks in the polarization current may appear for the surface with polarization enhanced. One peak corresponds to the reversal of the interior, and the other peak, the reversal of the surface polarization. The appearance of the surface peak is sensitive to the surface conditions and size. By way of contrast, we predict that it is possible to accelerate the switching process if surface conditions suppress surface polarization. Experimental study of the surface peak would therefore provide direct information on the surface conditions, such as polarization enhancement or suppression near the surface. Similar phenomena are expected to be observed in ferroelectric ultra-thin films.

The work of R.L.S. was supported by an ARC Large Grant. J.O. and K.H.C. would like to acknowledge the Malaysia government for the financial support they received through IRPA grant and Universiti Sains Malaysia. This work was also supported by the Center for Smart Materials of The Hong Kong Polytechnic University.
${ }^{1}$ J. F. Scott, Ferroelectric Memories (Springer-Verlag, Berlin, 2000).

${ }^{2}$ H. Uchida, N. Soyama, K. Kageyama, K. Ogi, and C. A. Paz de Araujo, Integr. Ferroelectr. 8, 41 (1997).

${ }^{3}$ C. S. Ganpule, S. Stanishevsky, Q. Su, S. Aggarwal, J. Mengailis, E. Williams, and R. Ramesh, Appl. Phys. Lett. 75, 409 (1999).

${ }^{4}$ M. Alexe, C. Harnagea, A. Visinoiu, A. Pignolet, D. Hesse, and U. Gösele, Scr. Mater. 44, 1175 (2001)

${ }^{5}$ M. Alexe, C. Harnagea, D. Hesse, and U. Gösele, Appl. Phys. Lett. 79(2), 242 (2001).

${ }^{6}$ S. Bühlmann, B. Dwir, J. Baborowski, and P. Muralt, Appl. Phys. Lett. 80(17), 3195 (2002).

${ }^{7}$ Y. Ishibashi, Integr. Ferroelectr. 2, 44 (1992).

${ }^{8}$ R. L. Stamps and B. Hillebrands, Appl. Phys. Lett. 75, 1143 (1999).

${ }^{9}$ M. A. Collins, A. Blumne, J. F. Currie, and J. Ross, Phys. Rev. B 19, 3630 (1979); J. F. Currie, A. Blumen, M. A. Collins, and J. Ross, ibid. 19, 3645 (1979).

${ }^{10}$ V. Y. Shur, Ferroelectric Thin Films: Synthesis and Basic Properties (Gordon and Breach, Amsterdam, 1996), p. 153.

${ }^{11}$ P. Ghosez and K. M. Rabe, Appl. Phys. Lett. 76(19), 2767 (2000).

${ }^{12}$ D. R. Tilley and B. Zeks, Solid State Commun. 49, 823 (1984).

${ }^{13}$ T. Mitsui, I. Tatzusaki, and E. Nakamura, An Introduction to the Physics of Ferroelectrics (Gordon and Breach, New York, 1976). 